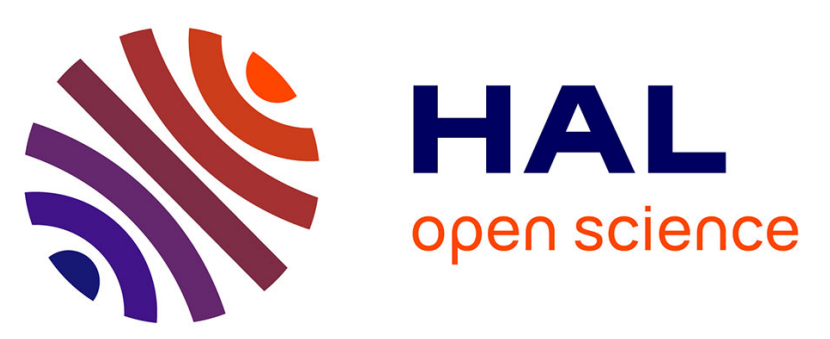

\title{
Modeling UV-Vis spectra of low dimensional materials using electrostatic embedding: the case of CdSe
}

\author{
Davide Luise, Liam Wilbraham, Frédéric Labat, Ilaria Ciofini
}

\section{To cite this version:}

Davide Luise, Liam Wilbraham, Frédéric Labat, Ilaria Ciofini. Modeling UV-Vis spectra of low dimensional materials using electrostatic embedding: the case of CdSe. Journal of Computational Chemistry, 2021, 42 (17), pp.1212-1224. 10.1002/jcc.26534 . hal-03297217

\section{HAL Id: hal-03297217 https://hal.science/hal-03297217}

Submitted on 23 Jul 2021

HAL is a multi-disciplinary open access archive for the deposit and dissemination of scientific research documents, whether they are published or not. The documents may come from teaching and research institutions in France or abroad, or from public or private research centers.
L'archive ouverte pluridisciplinaire HAL, est destinée au dépôt et à la diffusion de documents scientifiques de niveau recherche, publiés ou non, émanant des établissements d'enseignement et de recherche français ou étrangers, des laboratoires publics ou privés. 


\title{
Modeling UV-Vis spectra of low dimensional materials using electrostatic embedding: the case of CdSe
}

\author{
D. Luise ${ }^{1}$, L. Wilbraham ${ }^{2}$, Frédéric Labat ${ }^{1}$, Ilaria Ciofini ${ }^{1}$ \\ ${ }^{1}$ Chimie ParisTech, PSL University, CNRS, Institute of Chemistry for Life and Health Sciences, \\ Chemical Theory and Modelling Group, F-75005 Paris, France \\ ${ }^{2}$ School of Chemistry, University of Glasgow, University Avenue, Glasgow G12 8QQ, United \\ Kingdom
}

*Correspondence to: $\underline{\text { ilaria.ciofini@ chimie-paristech.fr and frederic.labat@ chimie-paristech.fr }}$

\begin{abstract}
We present a generalization of a self-consistent electrostatic embedding approach (SC-Ewald) devised to investigate the photophysical properties of 3D periodic materials, to systems in one- or two-dimensional (2D) reduced periodicity. In this approach, calculations are carried out on a small finite molecular cluster extracted from a periodic model, whilst the crystalline environment is accounted for by an array of point charges which are fitted to reproduce the exact electrostatic potential (at ground or the excited state) of the infinite periodic system. Periodic density functional theory (DFT) calculations are combined with time dependent DFT calculations to simulate absorption and emission properties of the extended system under investigation. We apply this method to compute the UV-Vis. spectra of bulk and quantum-confined OD quantum dots and 2D extended nanoplatelets of $\mathrm{CdSe}$, due to their relevance as sensitizers in solar cells technologies. The influence of the size and shape of the finite cluster model chosen in the excited state calculations was also investigated and revealed that, although the long-range electrostatics of the environment are important for the calculation of the UV-Vis, a subtle balance between short- and long-range effects exists. These encouraging results demonstrate that this self-consistent electrostatic embedding approach, when applied in different dimensions, can successfully model the photophysical properties of diverse material classes, making it an attractive low-cost alternative to far more computationally demanding electronic structure methods for excited state calculations.
\end{abstract}




\section{Introduction}

A large effort has recently been devoted to devise new photovoltaic technologies which could potentially outperform silicon-based solar cells in terms of efficiency and fabrication costs [1]. In particular, photosensitization of wide band gap n-type semiconductors (SC) such as $\mathrm{TiO}_{2}$ has paved the way to the development of various sensitized solar cell (SSC) technologies. Different classes of sensitizers have been tested, such as organic or inorganic dye molecules [2;3], quantum-dots (QD) [4] or perovskites [5;6]. The resulting photo-electrochemical devices usually use a monolayer of photosensitive material adsorbed on a thin layer of nanocrystalline oxide to harvest light, while a photoinduced electron transfer successively takes place from the sensitizer to the SC. In the case of QD-sensitized solar cells (QDSC), inorganic II-VI semiconductor nanoparticles (such as CdSe, CdS, $\mathrm{CdTe}, \mathrm{PbS}$ and $\mathrm{PbSe}$ ) have been used as sensitizers as their size-dependent optical properties can be leveraged to target specific applications. The nanoscopic nature of these particles induces a quantum confinement of the exciton wave function, increasing the nanoparticle's bandgap with decreasing size [7-8]. Furthermore, the nanocrystallites can generate multiple electron-hole pairs upon absorption of a single photon, which, in principle, allows one to exceed the Shockley-Queisser limit. Among the possible inorganic II-VI semiconductors for QDSC application, CdSe has been a material of choice. CdSe nanocrystallites can be readily obtained via colloidal synthesis both in aqueous and organic media [8;9], with various shapes and dimensions depending on the surfactant molecules used during synthesis, leading especially to zero dimensional (0D) quantum dots (QD) [7-8], 1D nanorods and 2D nanoplatelets (NPL)[10], with 1D and 2D objects having dimensions outside of the nanoscale range. In particular, QD are 3D confined spherical nanoparticles with typical diameters in the range of 2-10 nm, whereas CdSe NPL are 2D extended nanosheets confined due to their finite thickness along the normal to a well-controlled exposed plane at the experimental level [10]. Although both systems therefore present photophysical properties which can be tuned according to their dimensions and to the application targeted, CdSe NPL present some advantages over QD, including sharper photoluminescence, higher quantum yield and larger absorption cross section. $[11 ; 12 ; 13]$ They can also be easily synthesized by atomic deposition methods from monodisperse colloidal solutions of nanosheets, with a finely controlled thickness, layer by layer with atomic precision along the normal to the exposed (100) plane [10;14]. In addition, their excitonic and charge carrier properties can also be easily tuned by varying the passivating ligands during the synthesis. [15]

Due to their importance for photovoltaic applications, a better understanding of the light absorption properties of such objects is fundamental. However, the resulting CdSe sensitizers are either finite but composed of thousands of atoms as in the case of the QD species or extended 2D periodic objects 
such as NPL systems, preventing the use of the most advanced electronic structure methods to simulate and better understand their unique optical properties.

From a computational point of view, the study of excited state properties and phenomena such as the simulation of UV-Vis. spectra remains significantly challenging and computationally demanding when treating either large finite systems or extended periodic objects. For example, for the latter case, formulations have been proposed only recently, making use of either Gaussian-type Orbitals (GTO) $[16 ; 17 ; 18]$ or projector augmented wave [19] basis sets. In practice however, most studies addressing the calculation of the photophysical properties of such materials rely on extracting one part of the system, or cluster, and treating it at the time-dependent density functional theory (TD-DFT) level, which is well-established in the molecular field [see 20 for instance]. Further refinement of the cluster model can be performed to treat larger molecular aggregates by partitioning this molecular cluster into different layers to which different levels of theory are applied, either belonging to the quantummechanics (QM) or molecular mechanics (MM) fields, resulting in the so-called QM/MM [21] or QM/QM' [22; 23;24;25;26;27;28] techniques. In most cases however, the effect of the crystalline environment surrounding the extracted cluster is either absent or only partially addressed.

To simultaneously overcome the barrier of cluster size and retain a high-fidelity computational model, we recently proposed the SC-Ewald approach [29] to simulate the photophysical properties of 3D periodic materials, inspired by the original work of Derenzo et al. [30] which focused on the study of localized structural defects in ionic crystals. SC-Ewald is based on the concept of simulating the environment produced by a periodic system by an array of point charges, which are fitted to reproduce the exact electrostatic potential (at the ground or excited state) of an infinite periodic system. The photophysical properties of an infinite periodic solid can thus be computed at moderate computational cost by carrying out calculations on a molecular cluster treated at the QM level, while its surroundings are approximated by point charges to reproduce the electrostatic interaction of the extended system with the cluster. In addition, since the array of point charges can be self-consistently equilibrated with a given excited state of the molecular cluster, not only absorption but also emission spectra can be simulated. So far, this approach has been successfully applied to different phenomena occurring in 3D periodic systems such as excited-state intramolecular proton-transfer [31], aggregation induced emission [32] and mechanochromism [33]. A similar method has been lately developed and applied in the Crespo-Otero group (see for instance ref. 34). Here, we present an extension of the SC-Ewald approach to systems with reduced periodicity, that is systems that are periodic in one (polymers, helices, nanotubes) or two directions (surfaces). As a case study, we consider the application of this method to investigate both quantum-confined QD and NPL of CdSe, along with bulk CdSe. We note 
that, to the best of our knowledge, only one similar approach based on electrostatic embedding and applicable to periodic systems in one, two or three directions has already been proposed: the Periodic Electrostatic Embedded Cluster Method (PEECM) [35], although no applications to excited-state properties calculations have been reported so far.

The paper is structured as follows: in Section 2, the SC-Ewald approach is briefly recalled and its extension to systems with reduced periodicity is presented. Computational details are given in Section 3, while in Section 4, results obtained on CdSe bulk, QD and NPL are presented and discussed. Finally, in Section 5, some conclusions and perspectives of this work are drawn.

\section{The methods}

We first briefly recall the SC-Ewald approach originally developed for 3D periodic systems, before introducing modifications necessary to deal with 1D or 2D periodic systems. For 3D periodic systems, a large supercell obtained by replicating $\left(2 \mathrm{~N}_{\mathrm{x}}\right.$ x $2 \mathrm{~N}_{\mathrm{y}}$ x $\left.2 \mathrm{~N}_{\mathrm{z}}\right)$ crystallographic unit cells in the positive and negative directions about the origin of the crystallographic axis system is built, with atomic charges at the positions of the crystallographic atoms. This is further divided into three distinct zones: (i) an inner first zone $\left(\mathrm{Z}_{1}\right)$ containing a cluster of interest with $N_{1}$ atoms to be treated explicitly at the QM level; (ii) an intermediate second spherical zone $\left(\mathrm{Z}_{2}\right)$ with $N_{2}$ sites $\left(N_{2}>N_{1}\right)$, containing point charges held constant at values that are equal to the partial Mulliken atomic charges provided by periodic calculations, surrounding the first zone; (iii) an outer third zone $\left(\mathrm{Z}_{3}\right)$ with $N_{3}$ sites $\left(N_{3} \gg N_{2}\right)$ between the boundary of $\mathrm{Z}_{2}$ and the entire array formed by the $\left(2 \mathrm{~N}_{\mathrm{x}} \times 2 \mathrm{~N}_{\mathrm{y}} \times 2 \mathrm{~N}_{\mathrm{z}}\right)$ unit cells, containing variable point charges. The intermediate second zone with point charges held constant is introduced in order to avoid artificial over-polarization of the electron density in $\mathrm{Z}_{1}$ by the nearby point charges close to the QM boundary. In order to define the optimal array of charges, the following procedure is next applied:

1. The electrostatic potential of the infinite crystal lattice $\mathrm{V}_{\mathrm{E}}$ is computed at all sites of $\mathrm{Z}_{1}$ and $\mathrm{Z}_{2}$, using Ewald lattice formula [36]. For a 3D periodic system with $N$ atoms per unit cell with charges $q_{n}(n \in[1 ; N])$, a unit cell volume $V$ and a set of direct lattice vectors $\boldsymbol{T}$, the electrostatic potential at site with coordinates $\boldsymbol{x}_{m}$ is given by:

$$
\begin{aligned}
V_{E}^{3 D}\left(\mathbf{x}_{m}\right)= & \sum_{\mathbf{T}} \sum_{n=1}^{N^{\prime}} q_{n} \frac{\operatorname{erfc}\left(\xi\left|\mathbf{x}_{m}-\mathbf{x}_{n}+\mathbf{T}\right|\right)}{\left|\mathbf{x}_{m}-\mathbf{x}_{n}+\mathbf{T}\right|}+ \\
+ & \frac{4 \pi}{V} \sum_{k \neq \mathbf{0}} \frac{e^{-\frac{k^{2}}{4 \xi^{2}}}}{k^{2}} \sum_{n=1}^{N} q_{n} e^{-i \mathbf{k} \cdot\left(\mathbf{x}_{m}-\mathbf{x}_{n}\right)}-\frac{2 \xi}{\sqrt{\pi}} q_{m}
\end{aligned}
$$


where $N^{\prime}$ indicates that the term $(n=m, \boldsymbol{T}=\mathbf{0})$ is excluded from the real space sum, $\xi$ is a parameter controlling the relative decay of the real and reciprocal space sums and $\boldsymbol{k}$ form a discrete set of vectors in the reciprocal space.

2. The values of the point charges in $\mathrm{Z}_{3}$ are determined by solving a set of $\mathrm{N}_{3}$ linear simultaneous homogeneous equations to reproduce $\mathrm{V}_{\mathrm{E}}$ at the crystallographic sites in $\mathrm{Z}_{1}$ and $\mathrm{Z}_{2}$ :

$$
\sum_{i \neq k}^{N_{T}} \frac{q_{i}+\Delta q_{i}}{\left|\boldsymbol{r}_{\boldsymbol{k}}-\boldsymbol{r}_{\boldsymbol{i}}\right|}=V_{E}\left(\boldsymbol{r}_{\boldsymbol{k}}\right)
$$

where $k \in\left[1 ; N_{1}+N_{2}\right]$ and $N_{T}=N_{1}+N_{2}+N_{3}$ the total number of point charges in the three zones. Since $\mathrm{N}_{1}+\mathrm{N}_{2}<\mathrm{N}_{3}$, this is an underdetermined system for which we seek the minimum-norm solution to a real linear least squares problem:

$$
\min \|A X-B\|
$$

This solution selects the smallest possible variations $\Delta q_{i}$ of the charges in $\mathrm{Z}_{3}$. Additionally, the fitting procedure is constrained to produce a neutral charge array with a dipole moment of zero:

$$
\left\{\begin{array}{c}
\sum_{i>N_{1}+N_{2}}^{N_{T}} \Delta q_{i}=0 \\
\sum_{i>N_{1}+N_{2}}^{N_{T}} \Delta q_{i} \boldsymbol{r}_{i}=0
\end{array}\right.
$$

3. The quality of the charge-fitting is finally estimated by: (i) computing the root-mean square (RMS) error between the direct Coulomb sum potential and the exact Ewald potential for $\mathrm{N}_{\mathrm{R}}$ sites randomly chosen in $Z_{1}$ and $Z_{2}$. Typically, $N_{R}=1000$; (ii) evaluating the range of variation of charges in $\mathrm{Z}_{3}$. A wide variation indicates a failure at some point in the procedure. Furthermore we have tested that largest deviations of charges in $Z_{3}$ occurs at its border, that is the farthest from the QC cluster region (zone 1).

When generalizing this approach to $1 \mathrm{D}$ or 2D periodic systems, some modifications need to be introduced. Although no application to 1D systems is presented in this work, formulas and modifications required in this case are given below for completeness. In particular, supercells obtained by replicating $\left(2 \mathrm{~N}_{\mathrm{x}}\right)$ and $\left(2 \mathrm{~N}_{\mathrm{x}} \mathrm{x} 2 \mathrm{~N}_{\mathrm{y}}\right)$ crystallographic unit cells are built for $1 \mathrm{D}$ and $2 \mathrm{D}$ 
systems, respectively, and the site partitioning into three distinct zones mentioned above is carried out.

To determine $V_{E}\left(\boldsymbol{r}_{\boldsymbol{k}}\right)$ at all sites in $\mathrm{Z}_{1}$ and $\mathrm{Z}_{2}$, Ewald formulas in reduced periodicity are used [37]. More precisely, for a system periodic in the $x$ direction with $N$ atoms with charges $q_{n}(n \in[1 ; N])$ per unit cell, a lattice parameter $a$ and a set of direct lattice vectors $\mathbf{T}$, the electrostatic potential at site with coordinates $\mathbf{x}_{m}$ is given by:

$$
\begin{gathered}
V_{E}{ }^{1 D}\left(\mathbf{x}_{m}\right)=\sum_{\mathbf{T}} \sum_{n=1}^{N^{\prime}} q_{n} \frac{\operatorname{erfc}\left(\xi\left|\mathbf{x}_{m}-\mathbf{x}_{n}+\mathbf{T}\right|\right)}{\left|\mathbf{x}_{m}-\mathbf{x}_{n}+\mathbf{T}\right|} \\
+\frac{1}{a} \sum_{k_{1} \neq 0} \sum_{n=1}^{N} q_{n} e^{-i k_{1}\left(x_{m}-x_{n}\right)} K_{0}\left(\frac{k_{1}^{2}}{4 \xi^{2}}, \rho_{m n}^{2} \xi^{2}\right) \\
-\frac{1}{a} \sum_{n=1}^{N} q_{n}\left\{\gamma+\log \left(\rho_{m n}^{2} \xi^{2}\right)+E_{1}\left(\rho_{m n}^{2} \xi^{2}\right)\right\}-\frac{2 \xi}{\sqrt{\pi}} q_{m}
\end{gathered}
$$

where $K_{0}(u, v)$ is an incomplete modified Bessel function of the second kind defined as:

$$
K_{0}(u, v)=\int_{1}^{\infty} \frac{d t}{t} e^{-u t-\frac{v}{t}}
$$

and evaluated using an adaptive Romberg method, $E_{1}(v)$ is the exponential integral defined as:

$$
E_{1}(v)=\int_{1}^{\infty} \frac{1}{t} e^{-v t} d t=\int_{v}^{\infty} \frac{1}{t} e^{-t} d t
$$

$\gamma$ is the Euler Mascheroni constant, $\rho_{m n}=\sqrt{\left(z_{m}-z_{n}\right)^{2}+\left(y_{m}-y_{n}\right)^{2}}$ and $k_{1}$ forms a discrete set of $\mathbf{k}$ vectors in the reciprocal space.

Similarly, for a slab periodic in the $x$ and $y$ directions with unit cell area $A$ and a set of direct lattice vectors $\boldsymbol{T}$ :

$$
\begin{aligned}
& V_{E}^{2 D}\left(\mathbf{x}_{m}\right)=\sum_{T} \sum_{n=1}^{N^{\prime}} q_{n} \frac{\operatorname{erfc}\left(\xi\left|\mathbf{x}_{m}-\mathbf{x}_{n}+\mathbf{T}\right|\right)}{\left|\mathbf{x}_{m}-\mathbf{x}_{n}+\mathbf{T}\right|} \\
& +\frac{\pi}{A} \sum_{n=1}^{N} q_{n} \sum_{\bar{k} \neq 0} e^{-i \overline{\mathbf{k}} \cdot\left(\mathbf{r}_{m}-\mathbf{r}_{n}\right)} \frac{1}{\bar{k}} g\left(\bar{k}, z_{m}-z_{n}, \xi\right) \\
& -\frac{2 \sqrt{\pi}}{A} \sum_{n=1}^{N} q_{n}\left(\frac{1}{\xi} e^{-\xi^{2}\left(z_{m}-z_{n}\right)^{2}}+\sqrt{\pi}\left(z_{m}-z_{n}\right) \operatorname{erf}\left(\xi\left(z_{m}-z_{n}\right)\right)\right) \\
& -\frac{2 \xi}{\sqrt{\pi}} q_{m}
\end{aligned}
$$

where:

$$
g(\bar{k}, z, \xi)=e^{\bar{k} z} \operatorname{erfc}\left(\frac{\bar{k}}{2 \xi}+\xi z\right)+e^{-\bar{k} z} \operatorname{erfc}\left(\frac{\bar{k}}{2 \xi}-\xi z\right)
$$


and $\overline{\boldsymbol{k}}$ form a discrete set of vectors in the reciprocal space.

Finally, while the charge neutrality condition in $\mathrm{Z}_{3}$ (second equation in Eq. (3)) is still maintained, the dipole equation then becomes $\sum_{i>N_{1}+N_{2}}^{N_{T}} \Delta q_{i} x_{i}=0$ and $\sum_{i>N_{1}+N_{2}}^{N_{T}} \Delta q_{i} x_{i}+\sum_{i>N_{1}+N_{2}}^{N_{T}} \Delta q_{i} y_{i}=0$ for $1 \mathrm{D}$ and 2D periodic systems, respectively.

\section{Computational details}

\section{Ground state periodic calculations:}

All ground state periodic calculations were carried out at the DFT level with the Crystal17 code [3839], which solves self-consistently both Hartree-Fock (HF) and Kohn-Sham equations using atomcentered Gaussian orbitals as basis set. Based on a previous benchmark on the performances of different exchange-correlation functionals for CdSe bulk [40] performed by some of us, the global hybrid B3PW91 functional has been selected for all periodic calculations. Additional semi-empirical London-type dispersion corrections were employed for the optimization of CdSe bulk, considering the DFT-D3 scheme [41].

A double-zeta basis set augmented by one d polarization function, combined to the SBKJC pseudopotentials, have been used both for $\mathrm{Cd}$ and Se [42;43]. As a consequence, the following electrons were treated explicitly: $4 s^{2} 4 p^{6} 4 d^{10} 5 s^{2}$ for Cd and $4 s^{2} 4 p^{4}$ for Se. All ligands considered in the adsorption on the CdSe (100) surface were treated with all-electron basis sets, with the following contractions schemes: $(9 \mathrm{~s}, 3 \mathrm{p}, 1 \mathrm{~d}) \rightarrow[3 \mathrm{~s}, 2 \mathrm{p}, 1 \mathrm{~d}]$ for $\mathrm{C},(14 \mathrm{~s}, 6 \mathrm{p}, 1 \mathrm{~d}) \rightarrow[4 \mathrm{~s}, 3 \mathrm{p}, 1 \mathrm{~d}]$ for $\mathrm{O},(7 \mathrm{~s}, 1 \mathrm{p})$ $\rightarrow[3 \mathrm{~s}, 1 \mathrm{p}]$ for $\mathrm{H}$, and $(14 \mathrm{~s}, 8 \mathrm{p}, 1 \mathrm{~d}) \rightarrow[5 \mathrm{~s}, 4 \mathrm{p}, 1 \mathrm{~d}]$ for $\mathrm{S}$. [44]

The following tolerance criteria were set for the accuracy of Coulomb and exchange series: $10^{-7}$ as the overlap threshold of Coulomb integrals, $10^{-7}$ as penetration threshold of Coulomb integrals, $10^{-7}$ as overlap threshold for HF exchange integrals, $10^{-9}$ and $10^{-20}$ as pseudo-overlap for HF exchange series. An extra-large DFT integration grid was employed throughout, with 75 radial and 974 angular points. As a result of a comprehensive test carried out on each system on the convergence of the total energy with the increase of the k-points number, shrinking factors of 6,16 and 14 were selected for the NPL systems, Zinc Blende bulk and Wurtzite bulk respectively, thereby corresponding to 20, 145 and 192 k-points in the irreducible Brillouin zones of these systems. Full geometry optimizations (atomic coordinates and lattice parameters) were performed for all the periodic systems involved in the investigation, using default values [45] for convergence criteria.

CdSe NPLwere modeled as a zinc blende 2D periodic slab consisting of a film with a finite thickness exposing two free surfaces and composed of alternating layers of $\mathrm{Cd}$ and Se atoms parallel to the 
experimentally-exposed polar (100) crystallographic plane[46]. Since the experimental anionic ligands are known to link to Cd-rich surfaces, this surface was terminated by Cd layers on both top and bottom sides of the slab. Inorganic $\left(\mathrm{SH}^{-}\right.$and $\left.\mathrm{OH}^{-}\right)$and organic $\left(\mathrm{HCOO}^{-}\right)$anions were chosen as passivating ligands and the bidentate adsorption mode was considered following the results previously obtained in Ref [47]. HCOO was chosen to model the oleic acid since the long alkyl chain does not influence the electronic and optical properties of the ligand/nanoplatelet interface [47].

\section{Embedded excited-state calculations:}

All excited states calculations were performed at the TD-DFT level using a development version of the Gaussian code[48], considering the long-range corrected PBE (LC-PBE) functional[49;50]. combined to the double-zeta LANL2DZ basis set along with associated effective core potentials for all atoms $(\mathrm{Cd}, \mathrm{Se}, \mathrm{C}, \mathrm{H}, \mathrm{O}, \mathrm{S})$. LC-PBE belongs to the class of range-separated functionals, which have been developed to overcome some of the TD-DFT limitations originating from standard DFT when dealing with long range interactions. In particular, standard global hybrid functionals fail to correctly reproduce through-space particle-hole charge transfer interactions (arising upon electron excitation) due to the incorrect description of the asymptotic tail of the exchange potential at long range. On the other hand, long-range corrected functionals, including a variable percentage of $\mathrm{HF}$ like non local exchange depending on electron-electron distance, recover the correct asymptotic behavior of the exchange potential thereby allowing to better describe electron excitations of charge transfer character $[49 ; 50]$.

All clusters used for excited-state calculations were extracted from the optimized structures obtained with periodic boundary conditions, either in three or two directions, for the bulk and QD models and for the slab models, respectively. Point charges used for the electrostatic embedding were determined according to the procedure described in Section 2. All spectra presented below were obtained by convoluting computed vertical transitions with Gaussian functions with half-width of $0.01 \mathrm{eV}$.

In addition, in order to more quantitatively estimate the spatial extent of a photo-induced charge transfer, the $\mathrm{D}_{\mathrm{CT}}$ index has been used. Developed by some of us [51], it quantifies the length of the hole-electron separation due to a charge transfer excitation computed as the distance of the hole and electron charge density distributions baricenters ( $\mathrm{R}+$ and $\mathrm{R}-$, respectively).

$$
D_{C T}=\left|R_{+}-R_{-}\right|
$$

More details and examples of application can be found in ref [52;53]. 


\section{Results and Discussions}

To the best of our knowledge, there have been no previous attempts to model the excited state properties of CdSe bulk. On the other hand, several studies can be found in the literature of modeling the photophysical properties of CdSe quantum dots [54-63]. These works usually apply TD-DFT on bare and/or ligand-saturated nano-clusters composed by $\mathrm{Cd}$ and Se atoms [54, 55, 56]. This protocol has been used to model in particular the effect of the various capping molecules (e.g. $\mathrm{OPMe}_{3}, \mathrm{NH}_{2} \mathrm{Me}$ ) and of the solvent on the optical properties (such as UV-Vis. Absorption and emission spectra) of CdSe nanoparticles [54]. However, this approach is computationally limited by the size of the cluster, and has been applied to model CdSe nanoclusters composed by up to tens of atoms. Recently, Nadler and Sanz [57] simulated the optical spectra of CdSe nanoclusters both naked and capped with ligands composed of up to 68 atoms, using a real time TD-DFT approach (RT-TDDFT)[57]. They showed that RT-TDDFT can successfully reproduce the absorption spectrum of CdSe quantum dots at low computational cost. Consequently, they indicate the RT-TDDFT approach is more efficient for modeling medium- to large-sized systems than conventional linear-response TD-DFT implementations. However, this method's main drawback is that it does not allow for the assignment of the spectral features. As an alternative to first principles calculations, tight-binding models have also been used to model the size-dependence of the optical band-gap of CdSe QD [58, 59, 60, 61]. More recently, many-body perturbation theory calculations have also proved to perform well when applied to investigate the excitonic effects in CdSe nanocrystals, predicting optical gaps in good agreement with experiment[62]. On the other hand, concerning CdSe NPL, few theoretical works have been reported so far [62,63], all of them using a tight-binding approach [62], eventually combined [63] with Configuration Interaction to simulate the excitonic properties of NPLs.

In the present work, we propose a computational protocol to model the excited state properties of both extended systems (such as CdSe bulk or 2D CdSe NPLs) and finite size nano-clusters, relying on the same theoretical approach based on DFT and TD-DFT. By using the SC-Ewald methodology we were able to calculate vertical excitation energies and reproduce the UV-Vis. absorption spectra of the systems under investigation at low computational cost.

In this section, we will first discuss the results obtained on CdSe bulk, considering two phases: zinc blende and wurtzite. Next, CdSe systems with lower dimensionalities will be described, starting with QD of increasing sizes followed by NPL. For this latter, in agreement with a previous study, a welldefined (100) polar orientation stabilized by three passivating anionic ligands will be considered. 


\subsection{CdSe bulk}

CdSe is a II-VI semiconductor which can be obtained at atmospheric pressure both in the hexagonal wurtzite $\left(\mathrm{P}_{3} \mathrm{mc}\right)$ and cubic zinc blende (F-43m) phases, although $\mathrm{CdSe}$ single crystals are experimentally known to preferentially grow in wurtzite form.[64]. In both cases, $\mathrm{Cd}$ and $\mathrm{Se}$ are tetrahedrally coordinated and the two phases differ only in their stacking sequences: zinc blende shows an $\mathrm{ABCABC}$ sequence along the [111] direction while wurtzite has an $\mathrm{ABABAB}$ one along the $c$ axis so that only the third nearest neighbor configuration is different. For this reason, their electronic properties are very similar.[40]

Several studies can be found concerning the electronic and structural properties of bulk CdSe. [6568] In particular, a previous study by some of us [40] led to the development of a computational procedure, based on the use of global hybrid functionals, that serves as a good description of the structural and electronic features of CdSe bulk structures. The same protocol is applied here with the aim of choosing the best DFT approach. However, while in ref.40 a D2 dispersion correction scheme [69] was adopted in combination with each functional tested, we employed here the D3 [70] version implemented in Crystal17 thereby providing slightly different numerical results but the same qualitative description. As reported in Table SI.1 both optimized lattice parameters and computed band gaps obtained at the B3PW91-D3 level for both phases are in an overall very good agreement with the experimental data and previous literature data [40]. In particular, the maximal errors on the lattice parameters are of -0.016 and $-0.005 \AA$ for the wurtzite and zinc blende phases, respectively, while the corresponding errors on the band gaps are of +0.278 and $+0.137 \mathrm{eV}$, therefore validating the chosen DFT model for periodic calculations. As expected, similar Densities of States (DOS) are obtained for the two phases as reported in Figure SI.1: a sharp contribution of $\mathrm{Se}_{4 \mathrm{sp}}$ orbitals around $-13 \mathrm{eV}$, followed by a broader one around $-8 \mathrm{eV}$ mainly due to $\mathrm{Cd}_{4 \mathrm{~d}}$ orbitals with a small contribution from the $\mathrm{Se}_{4 \mathrm{sp}}$ orbitals, indicating a $p$ - $d$ coupling characteristic of tetrahedrally-coordinated compounds. Indeed, in binary crystalline solids having $T_{d}$ site symmetry, the metal's $d$ orbitals have the same representation at $\Gamma$ than the anion's $p$ orbitals, due to the crystal field splitting imposing a break of the metal's $d$ states into two groups: the lower energy e $e_{\mathrm{g}}$ states and higher energy $\mathrm{t}_{2 \mathrm{~g}}$ state (coupling with the chalcogenide p orbitals). The top of the valence band (VB) is mainly due to $\mathrm{Se}_{4 \mathrm{sp}}$ contributions, with a non-negligible overlap with $\mathrm{Cd}_{5 \text { sp }}$ orbitals, indicating a partial covalent character of the Cd-Se bond in these bulk materials. This is further confirmed by the analysis of the computed Mulliken atomic charges, with values of \pm 0.381 and \pm 0.383 e| for the two species in the wurtzite and 
zinc blende phases, respectively. On the other hand, the bottom of the conduction band (CB) is mainly dominated by $\mathrm{Cd}_{5 \mathrm{sp}}$ orbitals, with a small overlap with $\mathrm{Se}_{4 \mathrm{sp}}$ contributions.

As mentioned above, to simulate the UV-Vis. spectra of the two CdSe bulk phases considered, the SC-Ewald approach was employed. In the case of Zinc Blende, its primitive unit cell with 2 atoms was chosen as the quantum cluster (QC) for all SC-Ewald calculations. A (6x6x6) supercell was built around the QC, accounting for a total of 3460 point charges, with 2937 point charges in $Z_{3}$ and 523 in $Z_{2}$ (see Figure SI.2). The resulting charges produce a RMS deviation from the Ewald potential of the order of $0.1 \mu \mathrm{V}$ at all sites of $Z_{1}$ and $Z_{2}$ while the range of variation of charges in $Z_{3}$ is between $0.475|\mathrm{e}|$ and $+0.466 \mathrm{e} \mid$. Since the Zinc Blende polymorph is metastable at room temperature and pressure [64], we were unable to find suitable experimental UV-Vis. absorption spectra to compare with our computed results. However, our calculations reveal that Zinc Blende should not absorb in the visible region (see Figure 1a). Indeed, the computed absorption spectrum presents the first intense electronic transition ( $\mathrm{f}=0.36 \mathrm{a} . \mathrm{u}$ ) at $320 \mathrm{~nm}$. This transition corresponds mainly to a one electron excitation from the HOMO-2 to the LUMO involving $\mathrm{Cd} s$ and Se $p$ orbitals with an associated $\sigma \rightarrow \sigma^{*}$ character. The associated $\mathrm{D}_{\mathrm{CT}}$ index $(2.955 \AA)$ confirms its weak charge transfer character.
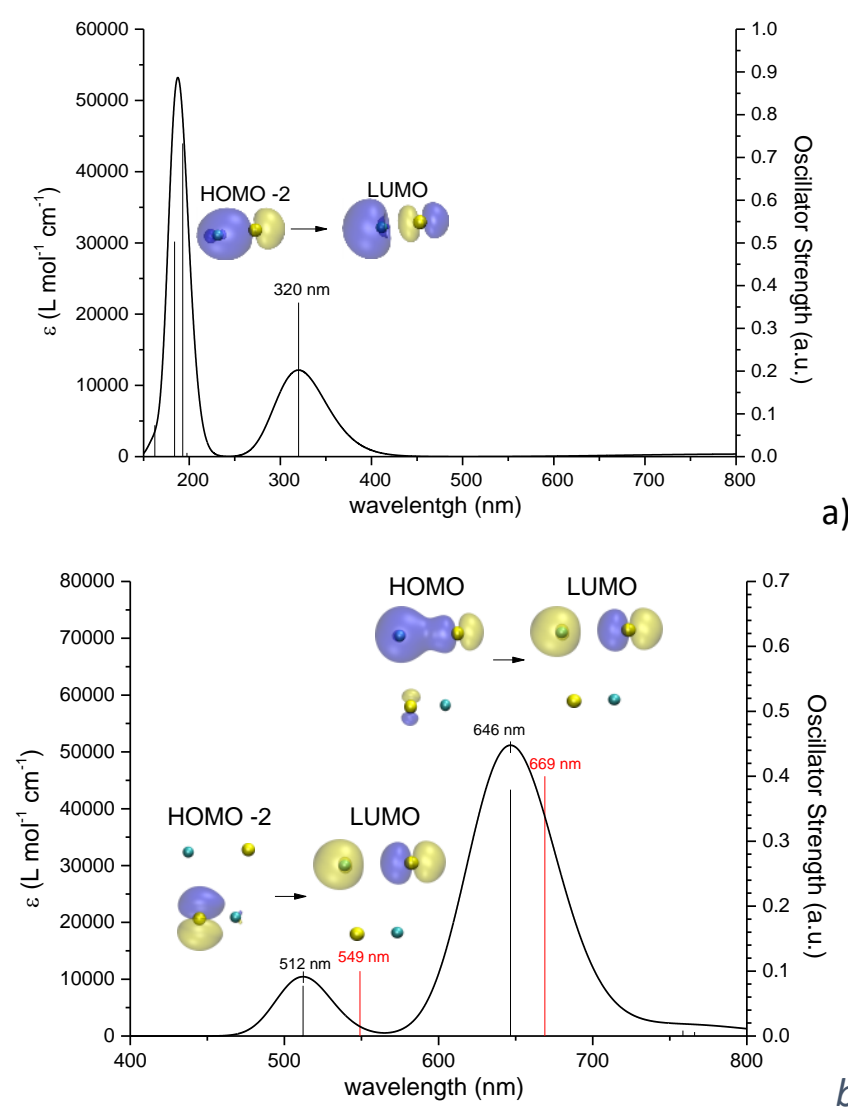

b)

Figure 1 (a) Computed UV-Vis. absorption spectra of CdSe Zinc Blende bulk. The molecular orbitals involved in the electronic transitions associated to the first absorption band are also plotted. (b) 
Computed UV-Vis. absorption spectra and experimental absorption band maxima positions (red vertical sticks, Ref. [72]) of CdSe wurtzite bulk. The molecular orbitals involved in the electronic transitions of each band are also plotted. Se and Cd atoms are depicted in yellow and cyan, respectively.

Following an analogous procedure, the Wurtzite phase UV-Vis. Absorption spectrum was also simulated. In this case, the primitive unit cell composed of 4 atoms ( $2 \mathrm{Cd}$ and $2 \mathrm{Se}$ ), is surrounded by an array of 12302 point charges (497 of which belonging to $Z_{2}$, see Figure SI.3) built from a (8x8x6) supercell. A RMS deviation from the Ewald potential of about $0.2 \mu \mathrm{V}$ at all sites of $\mathrm{Z}_{1}$ and $\mathrm{Z}_{2}$ was obtained, while $\mathrm{Z}_{3}$ charges ranged between -0.410 le $\mid$ and $+0.430|\mathrm{e}|$.

In Figure 1b the experimental absorption maxima (reported as sticks) and the computed spectra are reported. Experimentally CdSe wurzite bulk is characterized by two main absorption bands with associated absorption maxima at $669 \mathrm{~nm}(1.85 \mathrm{eV})$ and $549 \mathrm{~nm}(2.26 \mathrm{eV})$. The computed spectrum is in overall very good agreement with the experiments, with two bands computed at $647 \mathrm{~nm}$ (1.92 $\mathrm{eV})$ and $512 \mathrm{~nm}(2.42 \mathrm{eV})$. From the analysis of the orbitals involved in the vertical transitions responsible for the simulated bands, the lowest-energy band is associated to an intense electronic transition ( $\mathrm{f}=0.38$ a.u.) mainly corresponding to a one electron HOMO-LUMO excitation involving $\mathrm{Cd} s$ and Se $p$ orbitals with an associated $\sigma \rightarrow \sigma^{*}$ character. This is clearly consistent with the computed DOS reported in Figure SI.1 showing a combined contribution of the $s$ and $p$ orbitals of both Cd and Se atoms at the top of the VB and at the bottom of the CB. On the other hand, the second band computed at $512 \mathrm{~nm}$ arises from an electronic transition mainly corresponding to a one electron excitation from the HOMO-2 to the LUMO showing a $n \rightarrow \sigma^{*}$ character and less intense than the first one ( $\mathrm{f}=0.08$ a.u.). This transition shows a more pronounced charge transfer character than the first one, as quantified by the associated CT distances of $2.972 \AA$ for the $647 \mathrm{~nm}$ transition and 5.145 A for the $512 \mathrm{~nm}$ one. Indeed, in this latter case the hole-orbital is localized on one of the two selenium atoms, whereas the electron is delocalized over the other selenium and the nearby $\mathrm{Cd}$ atom present in the unit cell.

Overall, for CdSe bulk, a good qualitative and quantitative agreement of computed data with available experimental data has been obtained. In particular, for the UV-Vis. spectra simulation, the electrostatic embedding approach chosen here allowed to recover both short- and long-range electrostatic interactions at low computational cost, by considering a finite set of well-chosen point charges to reproduce the electrostatic effect of the infinite crystalline environment.

\section{$4.2 \mathrm{CdSe} Q D$}

To evaluate the performances of the SC-Ewald approach when applied to quantum-confined systems such as QD, the size-dependent UV-Vis. absorption spectra of CdSe QD was investigated, 
considering both zinc blende and wurtzite QDs. Since experimental QDs are known to be spherical with large sizes (in the 2-10 $\mathrm{nm}$ range) here, a 3D electrostatic embedding will be added around the cluster considered. Indeed, since the experimental QDs investigated here contain thousands of bulklike ordered atoms, their optical properties are simulated as being affected by the electrostatics produced by a bulk-like system. Additionally, since these two phases crystallize into the F-43m and $\mathrm{P}_{3} \mathrm{mc}$ space groups belonging to the cubic and hexagonal crystal systems, respectively, we also explored the impact of the asymmetry of the central QC on computed UV-Vis. spectra. This is here investigated by constructing $\mathrm{QC}$ of increasing sizes along a given lattice direction and verifying that the computed spectra are identical for symmetry-equivalent directions.

\section{a) Zinc Blende QD}

To first investigate if our model can recover the symmetry of the QC on the computed spectra, we extracted for each unit cell direction three different QC with 6,10 and 14 atoms (see Figure 2), by expanding the zinc blende unit cell along the three unit cell vectors, based on the optimized structure obtained with periodic boundary conditions presented in Section 4.1. In the following, in order to distinguish among all the QC, we will indicate by $\mathrm{x}-\mathrm{QC}$ the quantum cluster obtained by expanding the unit cell along the $\mathrm{x}(\mathrm{a}, \mathrm{b}$ or $\mathrm{c}$ ) lattice parameter. Each QC was surrounded by about 500 point charges in $\mathrm{Z}_{2}$ for a total of about 15000 embedding charges obtained from a (10x10x10) supercell of the crystallographic unit cell. For all cases considered, the computed RMS deviation from the Ewald potential at all sites of $\mathrm{Z}_{1}$ and $\mathrm{Z}_{2}$ was below $0.5 \mu \mathrm{V}$. Computed absorption spectra are reported in Figures SI.4-SI.6. It is clear that, for a given QC size, the computed spectra are independent from the expansion direction, nicely recovering the cubic symmetry of the zinc blende phase and highlighting the isotropic surroundings of the QC in such a material.

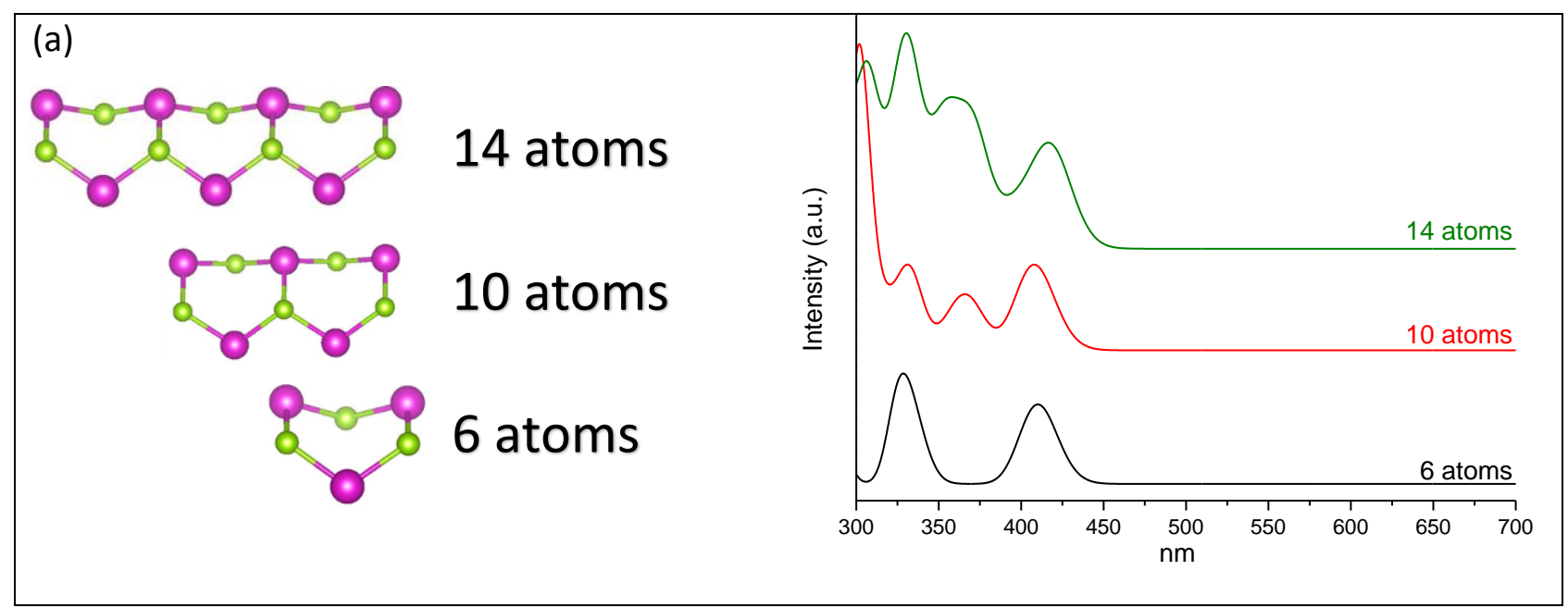


(b)
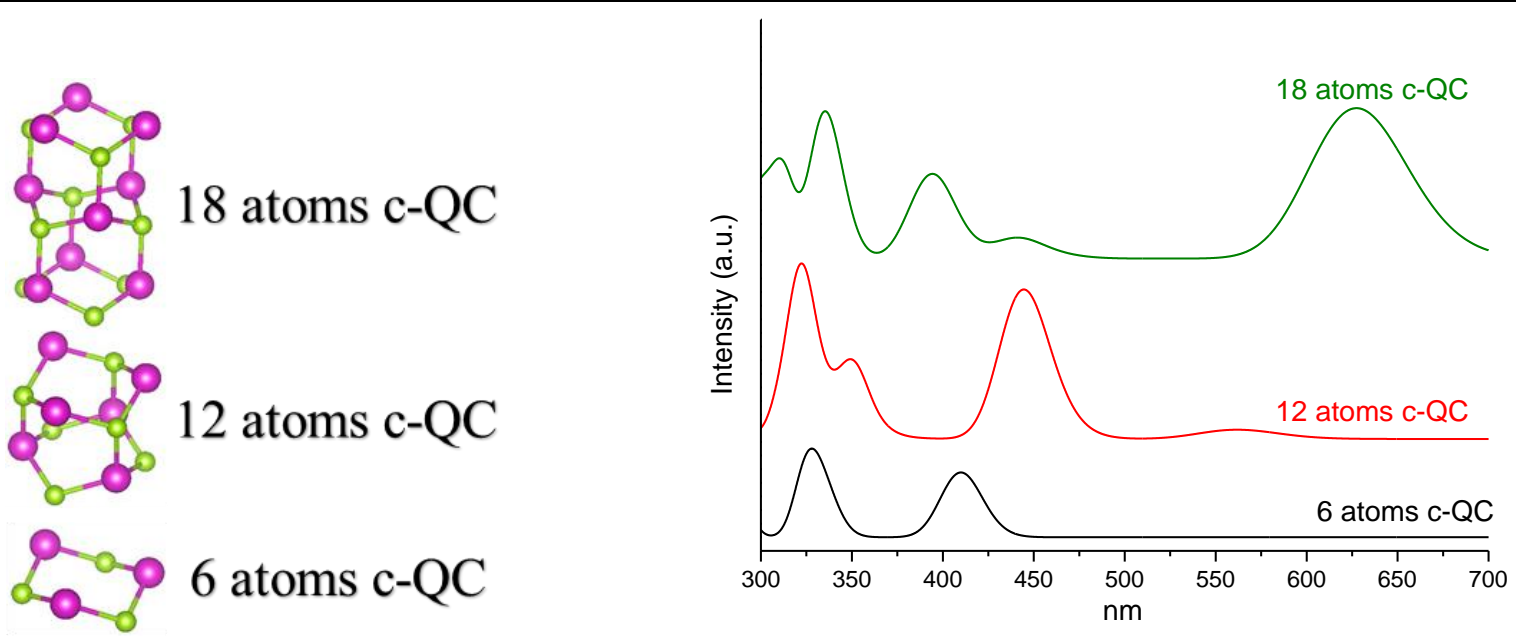

Figure 2: a) QC used for the zinc blende phase along all directions and for the a and $b$ directions of wurzite (left), with corresponding UV-Vis. Absorption spectra (right). B) QC used wurtzite along the c direction (left), with corresponding UV-Vis. Absorption Spectra (right).

Since, as expected, all computed UV-Vis. spectra are identical for a given QC size no matter which direction is chosen to expand the QC, in the following, we focus on a-QC only, that is QC expanded along the $a$ direction. Moreover, since experimental spectra of CdSe zinc blende QD are available for spherical QD with diameters between 12 and $23 \AA$, three additional a-QD with 18, 22 and 26 atoms, respectively were considered. A comparison between simulated and experimental spectra is provided in Figure 3, while a detailed analysis of band positions and band shifts is provided in Table SI.2.

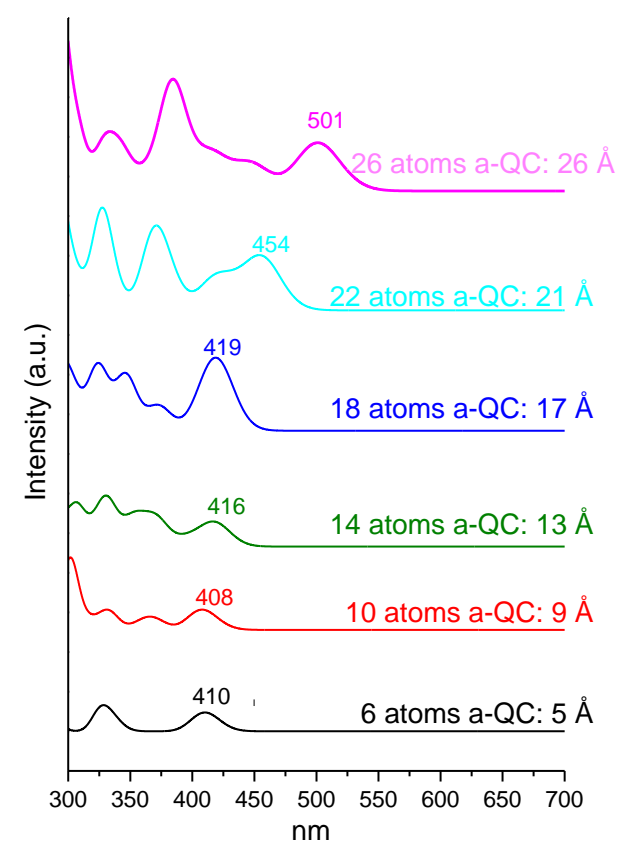

(a)

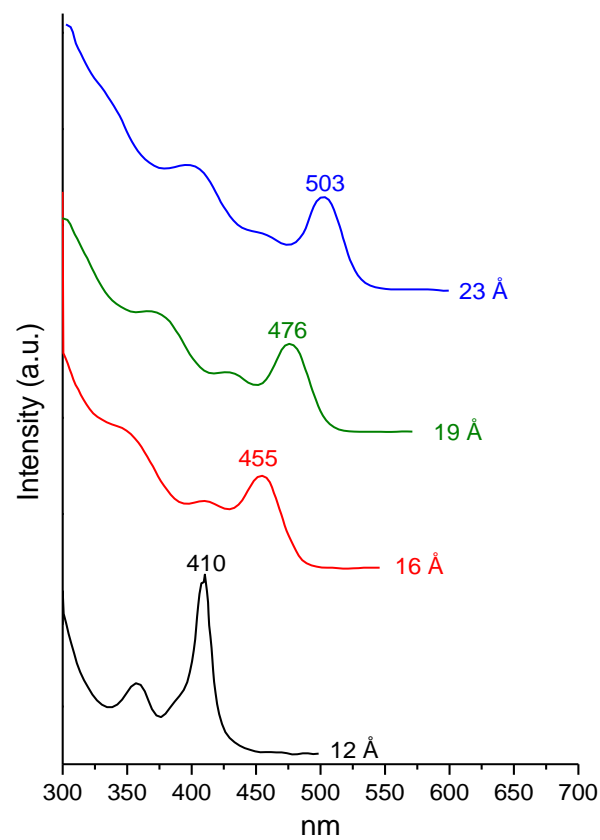

(b)

Figure 3: (a) Computed and (b) experimental [8] UV-Vis. absorption spectra of embedded CdSe zinc blende QC of increasing sizes (computed and experimental spectra of QD having similar size 
are in the same color). The first absorption peak maximum value ( $\lambda_{\max }$ in $\mathrm{nm}$, see Table SI.2) is reported, along with the associated largest QC dimension.

Experimentally, a redshift of the first absorption peak is observed when increasing the QD dimension. This is qualitatively reproduced by the computed data, although a direct comparison between the computed and experimental data is difficult since the sizes considered are not identical in both cases. In particular, the QD sizes indicated in Figure 3 refer to the QD diameter in the case of the experimental data, while they correspond to the end-to-end length in the case of the modeled QC, which can then be considered as only a portion of the real quantum dots. Nevertheless, the two absorption peaks positions obtained experimentally for the smallest and the largest QD at $410 \mathrm{~nm}$ and $503 \mathrm{~nm}$ are well-reproduced by the computed data at 410 and $501 \mathrm{~nm}$, although calculations are underestimating the red shift with increasing size. However, we note that both the shape of the whole spectrum and the relative intensities of the different bands computed are in line with the experimental data, constituting encouraging results for the application of the SC-Ewald approach to the modeling of the photophysical properties of difficult systems such as real QD made of thousands of atoms.

On the other hand, looking at the spectra obtained on naked QD models (i.e. without the application of the SC-Ewald embedding), reported in Figure SI.7, broad low-lying (582-630 nm) first absorption bands whose intensities decrease with the QC size are obtained. More importantly, it is evident that the calculated spectra do not replicate the bathochromic trend with the QD size observed experimentally (see Figure 3b) and overall, their shape and peak position do not resemble the experimental ones.

To better understand the optical properties of QD nanoparticles, the nature of the electronic transitions contributing to the first absorption band have been further analyzed, selecting the 26 atoms QC as an example. As reported in Supporting Information (Figure SI.8) for this cluster the lowest-energy band located at $501 \mathrm{~nm}(2.47 \mathrm{eV})$ is dominated by one single bright transition $\left(\mathrm{S}_{0} \rightarrow \mathrm{S}_{1}\right)$ corresponding to a $\mathrm{HOMO} \rightarrow$ LUMO excitation of charge transfer character as indicated by a $\mathrm{D}_{\mathrm{CT}}$ value of $4.980 \AA$. The HOMO is mainly composed by Se $4 p$ orbitals while the LUMO is a combination of $\mathrm{Se} 4 \mathrm{p}$ and $\mathrm{Cd} 4 \mathrm{~s}$ orbitals. Overall, for all the QC models (except for the 6 atoms QC being too small), the first bright transition which is responsible for the absorption onset implies a charge transfer from Se $4 \mathrm{p}$ to $\mathrm{Cd} 4 \mathrm{~s}$ as it is evident from the analysis of the electron density difference between the first (in the case of 26 atoms, 14 atoms, 10 atoms and 6 atoms QC) or the second (in the case of 22 atoms and 18 atoms QC) excited state and the ground state (Figure SI.9).

This kind of transition is actually affected by the size of the QC considered, decreasing in energy with the increase of the particle size. In particular by analyzing the $\mathrm{D}_{\mathrm{CT}}$ values reported in Table 1, we can 
note an increase of the charge transfer extent associated to the first bright transition as the size of the QC model considered increases, thereby resulting in the experimentally observed redshift of the UVVis. spectra.

Table 1. $\mathrm{D}_{\mathrm{CT}}$ values, excitation wavelength and oscillator strength computed for the first bright transition in the case of CdSe QC models of different sizes.

\begin{tabular}{l|ccc}
\hline $\begin{array}{c}\text { Number of } \\
\text { atoms in the qc }\end{array}$ & DCT $(\AA) /$ states & $\begin{array}{c}\text { Wavelength } \\
(\mathbf{n m})\end{array}$ & $\begin{array}{c}\text { f } \\
(\text { a.u. })\end{array}$ \\
\hline $\mathbf{2 6}$ & $4.98\left(\mathrm{~S}_{0} \rightarrow \mathrm{S}_{1}\right)$ & 501 & 0.222 \\
$\mathbf{2 2}$ & $4.93\left(\mathrm{~S}_{0} \rightarrow \mathrm{S}_{2}\right)$ & 457 & 0.226 \\
$\mathbf{1 8}$ & $2.69\left(\mathrm{~S}_{0} \rightarrow \mathrm{S}_{2}\right)$ & 424 & 0.150 \\
$\mathbf{1 4}$ & $1.91\left(\mathrm{~S}_{0} \rightarrow \mathrm{S}_{1}\right)$ & 419 & 0.055 \\
$\mathbf{1 0}$ & $1.60\left(\mathrm{~S}_{0} \rightarrow \mathrm{S}_{1}\right)$ & 413 & 0.037 \\
$\mathbf{6}$ & $0.97\left(\mathrm{~S}_{0} \rightarrow \mathrm{S}_{1}\right)$ & 410 & 0.044 \\
\hline
\end{tabular}

b) Wurtzite QD

Following the strategy presented for the zinc blende case, to test the influence of the asymmetry of the QC on the simulated UV-Vis. spectra, in the case of Wurzite QDs, three QC with increasing sizes were first constructed, considering expansion directions along the $\mathrm{a}, \mathrm{b}$ and $\mathrm{c}$ directions. Models with 6, 10 and 14 atoms were built along the two equivalent $\mathrm{a}$ and $\mathrm{b}$ directions, resulting in equivalent structures to those shown in Figure 2a for zinc blende, while models with 6, 12 and 18 atoms were considered along the non-equivalent c direction, as shown in Figure $\mathbf{2 b}$. 


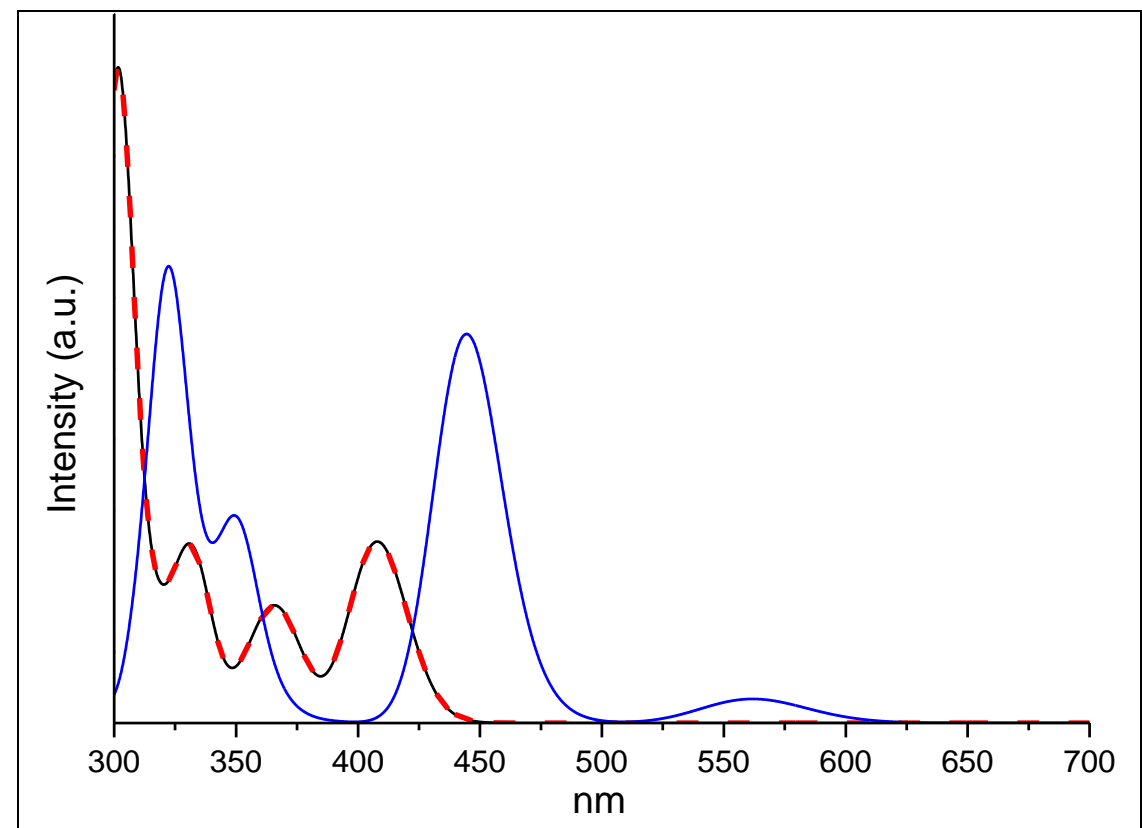

a)

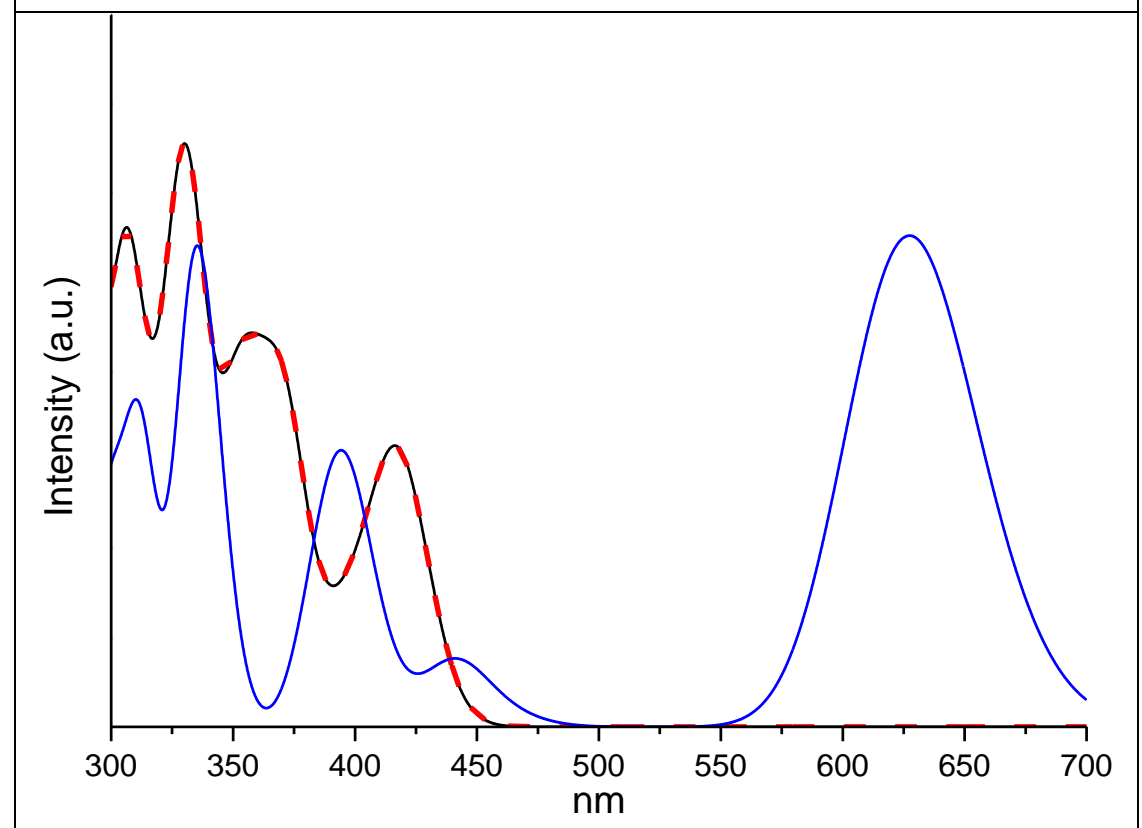

b)

Figure 4: Computed UV-Vis. Absorption spectra of Wurtzite with QC composed of (a) 10/12 atoms and (b) 14/18 atoms, and built by expansion along the a (solid black), $b$ (dashed red) and c (solid blue) lattice directions.

From the computed UV-Vis. spectra reported in Figure 4, it is clear that, as expected, the spectra obtained for the a- and b-QC are identical to the ones previously presented in the zinc blende case (see Figure SI.4-SI.6). On the other hand, c-QC data is significantly different which can be related to the 6-membered CdSe rings in chair-like conformation stacked one upon the other along this direction, thereby assuming a layered arrangement in this case, which is different from the zinc blende 
c-QC case (see Figure 3). Another notable difference can be observed upon increasing the QC size since new peaks appear at increasingly higher wavelengths. To better clarify this point, the largest cQC with 18 atoms was chosen for analysis of the transitions involved. In particular, the analysis of the lowest-energy band shows that the band maximum located at $627 \mathrm{~nm}(1.98 \mathrm{eV})$ is ascribed to a single electronic transition $\left(\mathrm{S}_{0} \rightarrow \mathrm{S}_{5}\right)$ of charge transfer character with an associated oscillator strength of 0.19 a.u. This transition stems from an electronic excitation involving the HOMO-4, composed mainly by Se $4 p$ orbitals, and the LUMO made up of $\mathrm{Cd} 4 \mathrm{~s}$ orbitals. The charge transfer character of the electronic transition was quantified by evaluating the associated $\mathrm{D}_{\mathrm{CT}}$ as done for the Zinc Blende case (see Figure SI.10). The 7.256 ̊ value obtained further confirms the marked charge transfer character associated to this electronic transition. We note that a similar investigation carried out on the 12 atoms c-QC led to similar results which are detailed in Figure SI.11 of the supporting material. Indeed, the first band at $525 \mathrm{~nm}(2.36 \mathrm{eV})$ is associated to a single electronic transition $\left(\mathrm{S}_{0} \rightarrow \mathrm{S}_{1}\right)$ of $\mathrm{HOMO} \rightarrow$ LUMO type and charge transfer character, with an associated $\mathrm{D}_{\mathrm{CT}}$ value of $4.013 \AA$.

Overall, the electrostatic embedding procedure considered here allowed to successfully recover important environmental effects impacting the photophysical properties of large systems such as QD, at low computational cost.

In particular, we were able to recover the size-dependent optical properties observed experimentally. Indeed, the bathochromic shift of the first absorption peak of CdSe QD of increasing sizes could be well-reproduced. In addition, for the CdSe zinc blende c-QC considered here, the hole-electron distance increases with the number of layers of CdSe rings, thereby indicating that peaks appearing at higher wavelengths originate from long-range electrostatic interactions, which weaken with the increase of the separation between the charge carriers. Finally, we note that when the environmental effects obtained by the SC-Ewald method are not included in the calculations, computed UV-Vis. spectra (see Figure SI.7) are unable to reproduce the experimental trend observed for the first absorption peak position, especially not showing the size-dependent optical shift typical of quantum confined nanoparticles.

\subsection{CdSe nanoplatelets (NPL)}

In this section, we explore the application of the 2D electrostatic embedding scheme to compute the UV-Vis. absorption spectra of polar CdSe NPL, passivated with different ligands. We also evaluate the effect of the quantum confinement by considering slab models with three different thicknesses along the non-periodic direction. Indeed, CdSe NPL are known to preferentially grow in the zinc blende phase, exposing the (100) polar orientation at the experimental level. The slab models considered are composed of alternating charged layers of $\mathrm{Cd}^{2+}$ and $\mathrm{Se}^{2-}$ ions, so that an N-layer slab 
consists of N/2 layers of Se atoms and N/2+1 layers of $\mathrm{Cd}$. The slab termination with $\mathrm{Cd}^{2+}$ cations and the stacking of charged planes along the normal to the exposed plane lead to dangling bonds breaking the structure's symmetry and creating a non-null dipole moment along the normal to the surface, indicating that the CdSe (100) slab model is a polar Type 3 slab model according to Tasker's classification [72]. Therefore, three anionic ligands $\left(\mathrm{OH}^{-}, \mathrm{SH}^{-}\right.$and $\left.\mathrm{HCOO}^{-}\right)$were employed to passivate under coordinated $\mathrm{Cd}$ atoms exposed at the surface, thereby ensuring the charge neutrality of the slab models and nullifying the dipole moment arising perpendicular to the surface, resulting in a stable (100) surface model. In the following, the three resulting passivated slab models will be referred to as CdSe.SH, CdSe.OH and CdSe.HCOO, respectively. The models of the CdSe.SH, $\mathrm{CdSe} . \mathrm{OH}$ and CdSe.HCOO slabs are shown in Figure 5. These models are equivalent to those already used by some of us in a previous work.[47]
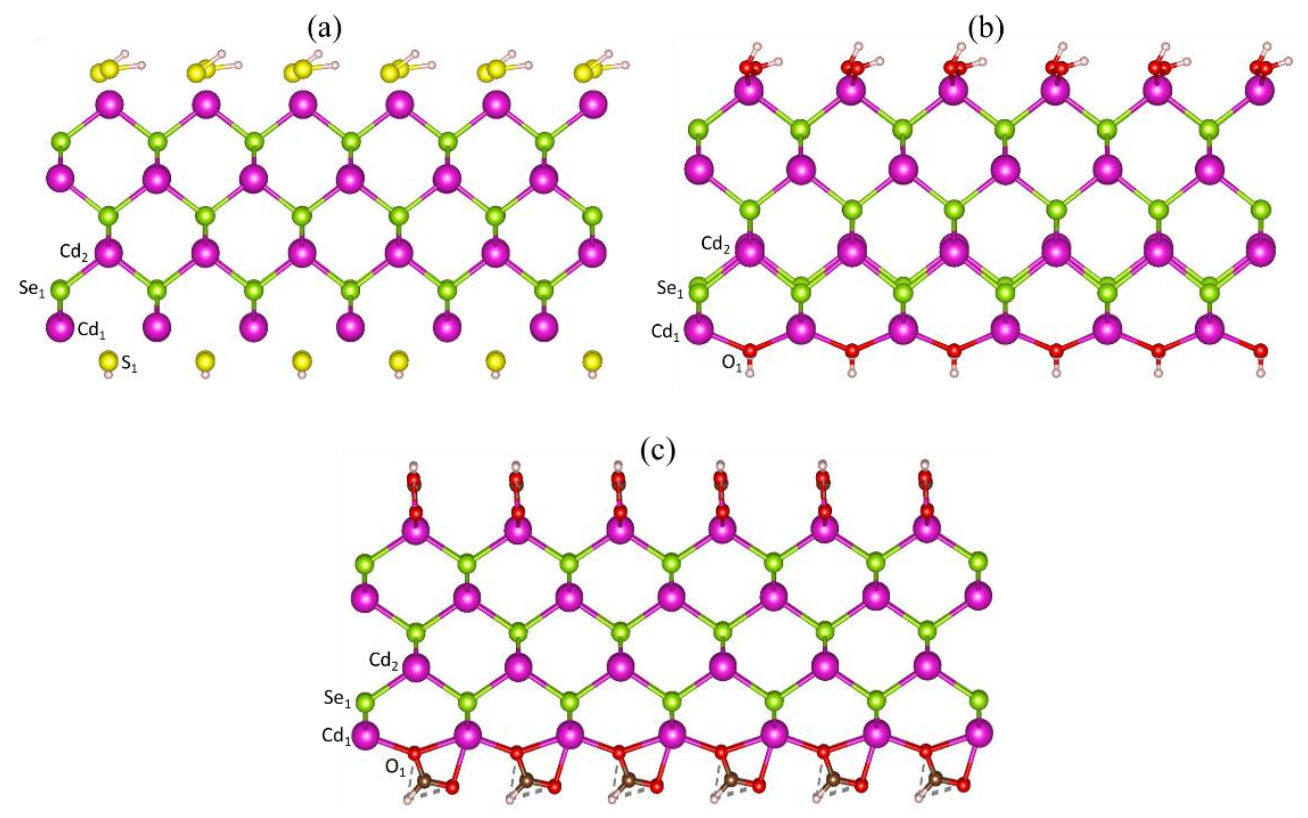

Figure 5: 7-layers CdSe zinc blende (100) slab model passivated with $\mathrm{SH}^{-}(a), \mathrm{OH}^{-}(b)$ and $\mathrm{HCOO}^{-}$ (c)ligands with 4 layers of $\mathrm{Cd}^{2+}$ (in violet) alternating with 3 layers of $\mathrm{Se}^{2-}$ (in green). White, yellow, brown and red balls correspond to $H, S, C$ and $O$ atoms respectively.

A 2D periodic geometry optimization of the resulting CdSe.SH, CdSe.OH and CdSe.HCOO slabs was first performed at the B3PW91-D3 level, by fully relaxing both lattice parameters and atoms, for slab models having 5, 7 and 9 layers that we refer to as 5L, 7L and 9L in the following. As a result of the geometrical optimization, as can be evidenced in Figure 5, in the case of the CdSe.SH slab model, sulfide ions occupy similar positions as the Se atoms in the inner layers, thereby respecting the tetrahedral arrangement of the CdSe structure. Indeed, the computed $\mathrm{S}_{1}-\mathrm{Cd}_{1}-\mathrm{Se}_{1}$ valence angle $\left(110^{\circ}\right.$, see Figure 5 for labeling) is very close to the $\mathrm{Cd}_{1}-\mathrm{Se}_{1}-\mathrm{Cd}_{2}$ one $\left(111^{\circ}\right)$. Furthermore, Cd-S distances are very similar $(2.598 \AA)$ to $\mathrm{Cd}-\mathrm{Se}(2.671 \AA)$ ones. Interestingly, the H-S-Cd-Se dihedral angles in 
the (001) plane are not always equal, with two alternating values of 68 and $35^{\circ}$. A very similar structural arrangement is computed for the $\mathrm{OH}^{-}$anions in $\mathrm{CdSe} . \mathrm{OH}$ models. Indeed, $\mathrm{OH}^{-}$is located on the surface between two adjacent $\mathrm{Cd}$ atoms, occupying similar positions as the inner Se atoms, thereby forming a perfect $\mathrm{O}_{1}-\mathrm{Cd}_{1}-\mathrm{Se}_{1}$ tetrahedral angle of $109^{\circ}$. Computed O-Cd distances $(2.305 \AA)$ are shorter than $\mathrm{Cd}$-Se ones (2.671 $\AA$ ), thereby suggesting a stronger interaction of the former compared to the latter. As already found for the CdSe.SH model, the H-O-Cd-Se dihedral angles found in the plane (001) take two alternating values of 95 and $49^{\circ}$. This can be related to steric repulsions which hamper hydrogens of two adjacent $\mathrm{OH}^{-}$(or $\mathrm{SH}^{-}$) anions to be too close. In the case of the CdSe.HCOO model, the $\mathrm{HCOO}^{-}$anions assume a bridging mode with one of the two oxygens bridging two adjacent $\mathrm{Cd}$ atoms. The bridging oxygen atoms are located in the same position as the oxygen in $\mathrm{CdSe} . \mathrm{OH}$ model, with the same $\mathrm{Cd}-\mathrm{O}(2.305 \AA)$ distance and the same $\mathrm{O}_{1}-\mathrm{Cd}_{1}-\mathrm{Se}_{1}\left(109^{\circ}\right)$ valence angle. Contrary to $\mathrm{CdSe} . \mathrm{SH}$ and $\mathrm{CdSe} . \mathrm{OH}$ models, due to the rigid planar configuration of the $\mathrm{HCOO}^{-}$anion, the C-O-Cd-Se dihedral angle is always equal to $125^{\circ}$. Table SI.3 reports computed bandgaps for all three models, together with the available experimental data. The decrease of the experimental bandgap when increasing the NPL thicknesses is qualitatively reproduced.

To simulate the UV-Vis. spectra of the NPL, the optimized unit cell was used as QC for the SC-Ewald embedding procedure: a (6x6) supercell was built from the slab unit cell, resulting in a total of about 6336 point charges, 300 of which belonging to $Z_{2}$, ensuring a RMS deviation from the Ewald potentials in $Z_{1}$ and $Z_{2}$ of about $4.7 \mu \mathrm{V}$ (see Figure SI.12). In Figure 6 the computed UV-Vis. spectra of CdSe.SH NPL of different thickness are compared to available experimental spectra.

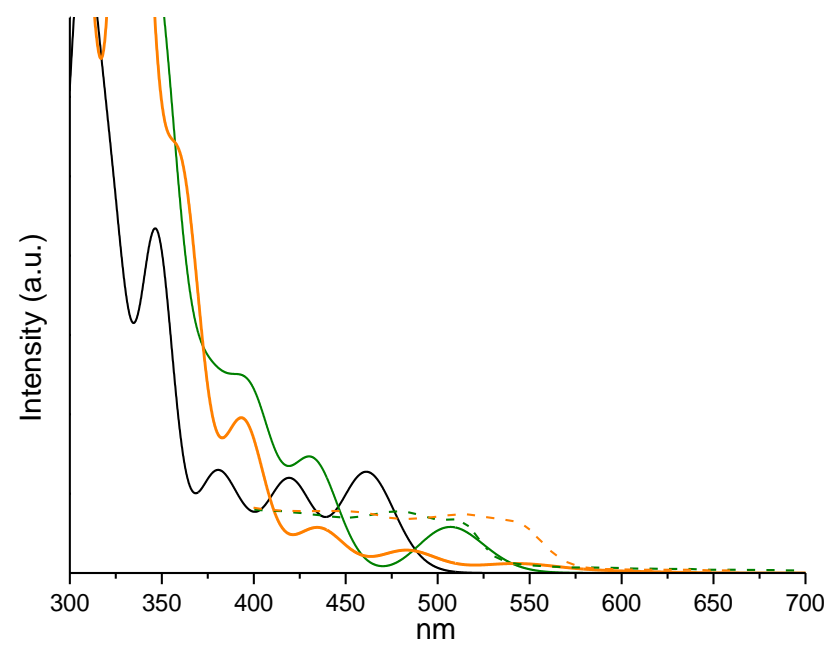

Figure 6: Computed (solid lines) and experimental (dashed lines) UV-Vis. absorption spectra of CdSe.SH NPL of different thickness (5 layers in black, 7 layers in green and 9 layers in orange). Experimental data from Ref. 74.

Experimentally, the absorption coefficient presents a step-like structure which can be interpreted by resorting to the model of the infinite one-dimensional quantum well. The first absorption band 
corresponds to the heavy hole - electron transition, the second one to the light hole - electron transition. From the computed NPL spectra, as expected, the first absorption peak undergoes a redshift upon increasing the number of layers in line with the experimental data. More quantitatively, from Table SI.4, an excellent agreement with the experimental data is obtained, both band positions and shifts being quantitatively reproduced when going from 7 to 9 layers, with an error of $\pm 0.01 \mathrm{eV}$.

The molecular orbitals involved in the electronic transitions contributing to the first absorption peak are shown in Table SI.5-8 of the supplementary material, for the three slab models with 5, 7 and 9 layers. The first band observed for the CdSe.SH $5 \mathrm{~L}$ and $9 \mathrm{~L}$ models arises from a single $\mathrm{S}_{0} \rightarrow \mathrm{S}_{1}$ transition involving essentially the HOMO and LUMO orbitals. On the other hand, two electronic transitions are responsible for the absorption peak observed in the case of CdSe.SH 7L NPL. The $\mathrm{S}_{0} \rightarrow \mathrm{S}_{1}$ transition is mainly due to the HOMO- $3 \rightarrow$ LUMO +5 excitation, while the $\mathrm{S}_{0} \rightarrow \mathrm{S}_{2}$ transition originates from the HOMO-21 $\rightarrow$ LUMO excitation.

The total and SH-projected DOS computed for the CdSe.SH models reported in Figure SI.13 indicate that $\mathrm{SH}^{-}$contributes both to the $\mathrm{VB}$ and $\mathrm{CB}$ edges in all cases, suggesting an overlap with the CdSe contribution, thus indicating a possible electron transfer between the passivating ligand and the CdSe NPL at the interface. This is further evidenced by the analysis of the electron density difference between the first excited state (which is responsible for the absorption onset observed in the NPL UV-Vis. spectra) and the ground state carried out for CdSe.SH 5L, 7L and 9L slabs (see Figure SI.14). Indeed, following the $S_{0} \rightarrow S_{1}$ electronic transition, the electron density redistribution is mainly localized at the SH-Cd interface. In fact, an electron depletion is observed right above the $\mathrm{S}$ atoms of the ligands, while an electron accumulation occurs close to the interfacial $\mathrm{Cd}$ atoms. The same conclusion holds for all the investigated CdSe.SH slabs, irrespective of the thickness, thereby indicating no effect of the increase of the number of $\mathrm{Cd}$ and Se layers on the electron transfer properties of the NPL (see Figure SI.15-SI.44).

Finally, to investigate the effect of the passivating ligand on the optical properties of the CdSe NPLs, computed absorption spectra of the 7-layers CdSe.SH NPL CdSe.OH and CdSe.HCOO NPL models are compared in Figure 7. 


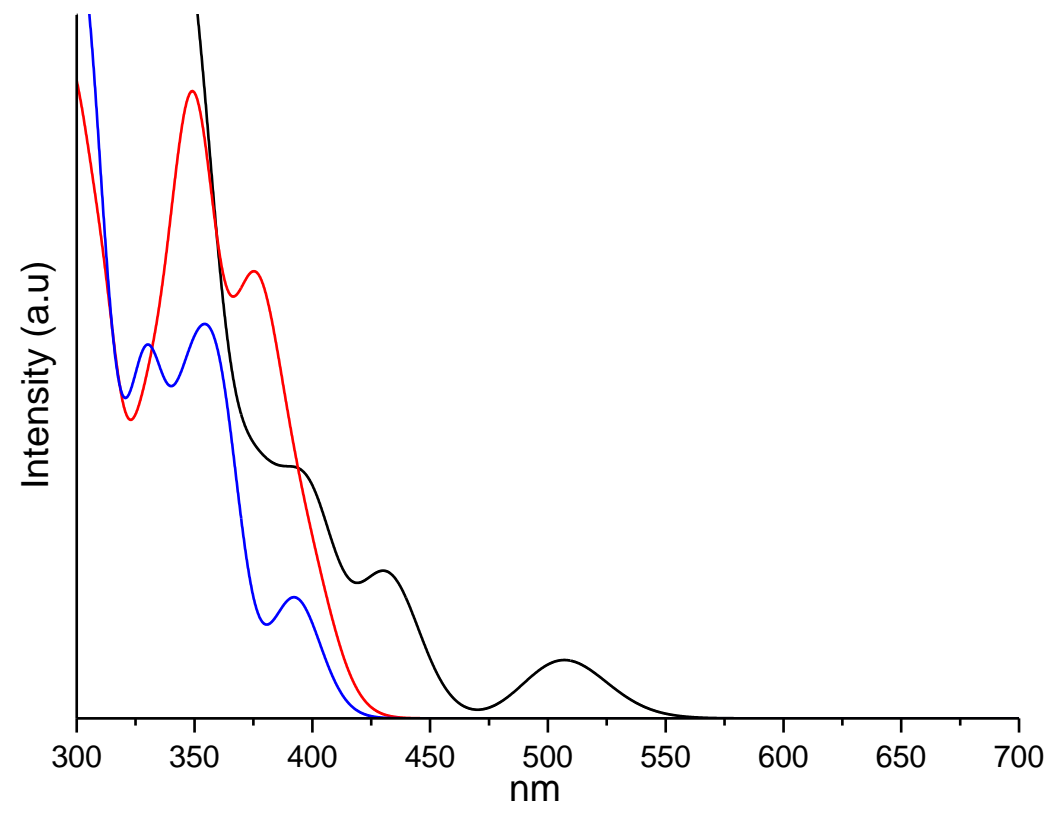

Figure 7: Computed UV-Vis. absorption spectra of CdSe.SH (black), CdSe.OH (red) and CdSe.HCOO (blue) 7 layers NPLs.

Compared to CdSe.SH, the absorption onset is blue-shifted for both $\mathrm{CdSe} . \mathrm{OH}$ and CdSe.HCOO. As a result, the absorption spectra of CdSe NPL passivated with $\mathrm{OH}^{-}$and $\mathrm{HCOO}^{-}$ligands cover a very tiny portion of the visible region, thereby suggesting a decrease in the light absorption properties of the CdSe NPL when these two latter ligands are used for passivation instead of $\mathrm{SH}^{-}$, with possible important consequences for photovoltaic application of such systems. In addition, it is interesting to note that the $\mathrm{S}_{0} \rightarrow \mathrm{S}_{1}$ electronic transitions computed at 403 and $393 \mathrm{~nm}$ for $\mathrm{OH}^{-}$and $\mathrm{HCOO}^{-}$, respectively, are characterized by weak intensity (low oscillator strengths) and are mainly localized on the core of the NPL (Table SI.9-SI.10). This is in clear contrast with the CdSe.SH case, where passivating $\mathrm{SH}$ - ligands were involved in the electronic transition, thereby indicating that $\mathrm{SH}^{-}$anions are better candidates than $\mathrm{HCOOH}^{-}$or $\mathrm{OH}^{-}$to passivate CdSe NPL sensitizers.

\section{Conclusions}

We have applied the SC-Ewald method to model the excited state properties of extended periodic objects by replicating their crystalline potential by means of an electrostatic embedding approach. As a result of the application of the SC-Ewald method, the UV-Vis. absorption spectra of the CdSe bulk (3D periodic), CdSe quantum dots (OD periodic) and CdSe NPL (2D periodic) were computed and showed very good agreement compared with available experimental UV-Vis. absorption spectra. Calculations showed that the choice of the quantum cluster (QC) was an important step and clearly affecting the results obtained. The results obtained with minimal cluster for the bulk phases allowed to recover the experimentally observed spectra at minimal computational cost. Our approach is also able to reproduce the main features of QD and, in particular, the size-dependent optical properties 
observed experimentally (ie the bathochromic shift of the first absorption peak of CdSe QD of increasing sizes). Of note this is not the case when using naked cluster, that is when excluding the charge embedding. Finally, complex 2D structures, such as CdSe nanoplatelets passivated with different ligands could be studied and the effect of the thickness of the platelets on the absorption spectra correctly reproduced. Overall, these results are encouraging for the use of embedding techniques when predicting photophysical properties of low dimensional materials at low computational cost.

\section{Acknowledgments}

D.L, I.C. and F.L. acknowledge the use of computational resources at Très Grand Centre de Calcul (TGCC) provided by GENCI through project A0050810135. I.C. gratefully acknowledges support from the European Research Council (ERC) for funding under the European Union's Horizon 2020 research and innovation programme (grant agreement No 648558, STRIGES CoG grant). I.C., F.L. and D.L. acknowledge support from the Marie Sklodowska Curie Innovative Training Network MSCA-ITN-2017 (grant number 765739 COSINE).

Data availability statement. The authors confirm that the data supporting the findings of this study are available within the article and its supplementary materials. 


\section{References:}

[1] El Chaar, L.; lamont, L. A.; El Zein, N. Renwewable and Sustainable Energy Reviews 2011, 15, 2165-2175

[2] O’Regan, B.; Grätzel, M. Nature 1991, 353, 737-740

[3] Hagfeldt, A.; Boschloo, G.; Sun, L.; Kloo, L.; Pettersson, H. Chem. Rev. 2010, 110, 6595-6663

[4] A. J. Nozik Phys. E. 2002, 14, 115-120

[5] Akihiro, K.; Kenshiro, T.; Yasuo, S.; Tsutomu. M. J. Am. Chem. Soc. 2009, 131, 6050-6051

[6] Lee, M. M.; Teuscher, J.; Miyasaka, T.; Murakami, T. N.; Snaith, H. J. Science 2012 338, 643647

[7] Neeleshwar, S; Chen, C. L.; Tsai, C. B. ; Chen, Y.Y. Physical Review B 2005, 71, 201307

[8] Murray, C. B.; Norris, D. J.; Bawendi, M. G. J. Am. Chem. Soc. 1993, 115, 8706-8715

[9] Kalasad, M. N.; Rabinal, M. K.; Mulimani, B. G. Langmuir 2009, 25, 21, 12729-12735

[10] Ithurria, S.; Dubertret, B. J. Am. Chem. Soc. 2008, 130, 16504- 16505

[11] Achtstein, A. W.; Schliwa, A.; Prudnikau, A.; Hardzei, M.; Artemyev, M. V.; Thomsen, C.; Woggon, U. Nano Lett., 2012, 12, 3151-7.

[12] Tessier, M. D.; Javaux, C; Maksimovic, I; Loriette, V.; Dubertret, B. ACS Nano, 2012, 6, 67518

[13] Yeltik, A.; Delikanli, S.; Olutas, M.; Kelestemur, Y.; Guzelturk, B.; Demir, H. V. J. Phys. Chem. C 2015, 119, 47, 26768-26775

[14] Ithurria, S. ; Tessier, M. D. ; Mahler, B. ; Lobo, R. P. S. M. ; Dubertret, B. ; Efros, A. L. Nature Materials 2011, 10, 936-941

[15] Szemjonov, A.; Pauporté, T.; Ithurria, S.; Lequeux, N.; Dubertret, B.; Ciofini, I.; Labat, F. RSC Adv. 2014, 4 (99), 55980-55989.

[16] Bernasconi, L.; Tomić, S.; Ferrero, M.; Rérat, M.; Orlando, R.; Dovesi, R.; Harrison, N. M. Phys. Rev. B 2011 83, 1-7

[17] Ferrero, M.; Rérrat, M.; Orlando, R.; Dovesi, R. J. Comput. Chem. 2008 29, 1450-1459

[18] Ferrari, A. M.; Orlando, R.; Rérat, M. J. Chem. Theory Comput. 2015 11, 3245-3258 
[19] Walter, M.; Häkkinen, H.; Lehtovaara, L.; Puska, M.; Enkovaara, J.; Rostgaard, C.; Mortensen, J. J. J. Chem. Phys. 2008 128, 244101

[20] Adamo, C.; Jacquemin, D. Chem. Soc. Rev. 2013 42, 845-856

[21] Jacquemin, D.; Mennucci, B; Adamo, C. Phys. Chem. Chem. Phys. 2011 13, 16987-16998

[22] Labat, F.; Ciofini, I.; Hratchian, H. P.; Frisch, M.; Raghavachari, K.; Adamo, C. J. Am. Chem. Soc. 2009 131, 14290-14298

[23] Le Bahers, T.; Di Tommaso, S.; Peltier, C.; Fayet, G.; Giacovazzi, R.; Tognetti, V.; Prestianni, A.; Labat, F. J. Mol. Struct.: Theochem 2010 954, 45-51

[24] Labat, F.; Ciofini, I.; Hratchian, H.; Frisch, M.; Raghavachari, K.; Adamo, C. J. Phys. Chem. C 2011 115, 4297-4306

[25] Labat, F.; Ciofini, I.; Adamo, C. J. Mater. Chem. 2012 22, 12205

[26] García, G.; Ciofini, I.; Fernández-Gómez, M.; Adamo, C. J. Phys. Chem. Lett. 2013 4, 12391243

[27] Presti, D.; Labat, F. ; Pedone, A.; Frisch, M. J.; Hratchian, H. P.; Ciofini, I.; Menziani, M. C.; Adamo, C. J. Chem. Theory Comput. 2014 10, 5577-5585

[28] Presti, D.;Labat, F. ; Pedone, A. ; Frisch, M. J.; Hratchian, H. P.; Ciofini, I.; Menziani, M. C.; Adamo, C. J. Comput. Chem. 2016 37, 861-870

[29] Wilbraham, L.; Adamo, C.; Labat, F.; Ciofini, I. J. Chem. Theory Comput. 2016 12, 3316-3324

[30] Derenzo, S. E.; Klintenberg, M. K.; Weber, M. J. J. Chem. Phys. 2000 112, 2074

[31] Wilbraham, L.; Adamo, C.; Labat, F.; Ciofini, I. J.Chem. Theory Comput. 2016, 12, 7, 33163324

[32] Presti, D.; Wilbraham, L.; Targa, C.; Labat, F.; Pedone, A.; Menziani, M. C.; Ciofini, I.; Adamo, C. J. Phys. Chem. C 2017, 121, 10, 5747-5752

[33] Wilbraham, L.; Louis, M.; Alberga, D.; Brosseau, A.; Guillot, R.; Ito, F.; Labat, F.; Métivier, R.; Allain, C.; Ciofini, I. Advanced Materials 2018, 1800817

[34] Rivera, M. ; Dommett, M. ; Sidat, A. ; Rahim, W. ; Crespo-Otero, R. J. Comput. Chem. 2020, 41, 1045-1058

[35] Burow, A. M.; Sierka, M.; Döbler, J.; Sauer, J. J. Chem. Phys. 2009 130, 174710 
[36] Ewald, P.P. Ann. Phys. 1921, 64, 253

[37] Tornberg, A. K. Adv. Comput. Math. 2016, 42, 227-242

[38] Dovesi, R.; Erba, A.; Orlando, R.; Zicovich-Wilson, C. M.; Civalleri, B.; Maschio, L.; Rerat, M.; Casassa, S.; Baima, J.; Salustro, S.; Kirtman, B. Wires Comput Mol Sci. 2018, 8, e1360

[39] Dovesi, R.; Saunders, V.R.; Roetti, C.; Orlando, R; Zicovich-Wilson, C. M.; Pascale, F.; Civalleri, B.; Doll, K.; Harrison, N. M.; Bush, I. J.; D’ Arco, P.; Llunell, M.; Causà, M.; Noël, Y.; Maschio, L.; Erba, A.; Rerat, M., Casassa, S. CRYSTAL17 User's Manual (University of Torino, Torino, 2017)

[40] Szemjonov, A.; Pauporté, T.; Ciofini, I.; Labat, F. Phys. Chem. Chem. Phys., 2014, 16, 23251

[41] Grimme, S.;Antony, J.; Ehrlich, S.; Krieg, S. “A consistent and accurate ab initio parametrization of density functional dispersion correction (dft-d) for the 94 elements $\mathrm{H}-\mathrm{Pu}$ ", $J$. Chem. Phys. 2010, 132, 154104

[42] Stevens, W. J ; Krauss, M. ; Basch, H. ; Jasien, P. G. Can. J. Chem. 1992, 70, 612

[43] Labello, N. P. ; Ferreira, A. M. ; Kurtz, H. A. J. Comput. Chem. 2005, 26, 1464

[44] Peintinger, M. F. ; Oliveira, D. V. ; Bredow, T., J. Comput. Chem., 2013, 34, 451

[45] CRYSTAL17 User's Manual Dovesi, R. ; Saunders, V. R. ; Roetti, C. ; Orlando R. ; ZicovichWilson, C. M. ; Pascale, F. ; Civalleri, B. ; Doll, k. ; Harrison, N. M. ; Bush, I. J. ; D’arco, Ph. ; Llunel, M. ; Causà, M. ; Noël, Y. ; Maschio, L. ; Erba, A. ; Rérat, M. ; Casassa, S.

[46] Ithurria, S. ; Tessier, M. ; Mahler, B. et al. Nature Mater 2011, 10, 936-941

[47] Szemjonov, A; Pauporté, T.; Ithurria, S.; Lequeux, N.; Dubertret, B.; Ciofini, I.; Labat, F. RSC Adv., 2014, 4, 55980

[48] Gaussian Development Version, Revision I.13, M. J. Frisch, G. W. Trucks, H. B. Schlegel, G. E. Scuseria, M. A. Robb, J. R. Cheeseman, G. Scalmani, V. Barone, G. A. Petersson, H. Nakatsuji, X. Li, M. Caricato, A. V. Marenich, J. Bloino, B. G. Janesko, R. Gomperts, B. Mennucci, H. P. Hratchian, J. V. Ortiz, A. F. Izmaylov, J. L. Sonnenberg, D. Williams-Young, F. Ding, F. Lipparini, F. Egidi, J. Goings, B. Peng, A. Petrone, T. Henderson, D. Ranasinghe, V. G. Zakrzewski, J. Gao, N. Rega, G. Zheng, W. Liang, M. Hada, M. Ehara, K. Toyota, R. Fukuda, J. Hasegawa, M. Ishida, T. Nakajima, Y. Honda, O. Kitao, H. Nakai, T. Vreven, K. Throssell, J. A. Montgomery, Jr., J. E. Peralta, F. Ogliaro, M. J. Bearpark, J. J. Heyd, E. N. Brothers, K. N. Kudin, V. N. Staroverov, T. A. Keith, R. Kobayashi, J. Normand, K. Raghavachari, A. P. Rendell, J. C. Burant, S. S. Iyengar, J. 
Tomasi, M. Cossi, J. M. Millam, M. Klene, C. Adamo, R. Cammi, J. W. Ochterski, R. L. Martin, K. Morokuma, O. Farkas, J. B. Foresman, and D. J. Fox, Gaussian, Inc., Wallingford CT, 2016

[49] Iikura, H. ; Tsuned, T. ; Yanai, T. ; Hirao, K. J. Chem. Phys. 2001, 115, 3540-3544

[50] Song, J. W. ; Hirosawa, T. ; Tsuned, T. ; Hirao, K. J. Chem. Phys. 2007, 126, 154105

[51] Garcìa, J. S. ; Maschietto, F. ; Campetella, M. ; Ciofini, I. J. Phys. Chem. A 2018, 122, 1, 375382

[52] Mashietto, F. ; Garcìa, J. S. ; Campetella, M. ; Ciofini, I. Journal of Computational Chemistry 2019, 40, 650-656

[53] Farré, Y. ; Maschietto, F. ; Föhlinger, J. ; Wykes, M. ; Planchat, A. ; Pellegrin, Y. ; Blart, E. ; Ciofini, I. ; Hammarström, L. ; Odobel, F. ChemSusChem 2020, 13, 1844-1855

[54] Sun, J. ; Zheng, X. ; He, H. ; Chen, X. ; Dong, B. ; Fei, R. Journal of Molecular Structure 1114 2016, 123-131

[55] Kilina, S. ; Ivanov, S. ; Tretiak, S. J. Am. Chem. Soc. 2009 131, 7717-7726

[56]Kilina, S. ; Velizhanin, K. A. ; Ivanov, S. ; Prezhdo, O. V. ; Tretiak, S. Acs Nano 2012, 6, 65156524

[57] Nadler, R. ; Sanz, J. F. Theor Chem Acc 2013, 132, 1342

[58] Lippens, P. ; Lannoo, M. Phys. Rev. B 1990, 41, 6079

[59] Lippens, P. ; Lanno, M. Mater. Sci. Eng. B. 1991, 9, 485-487

[60] Albe, V. ; Jouanin, C. ; Bertho, D. Phys. Rev. B 1998, 58, 4713

[61] Pérez-Conde, J. ; Bhattacherjee, A Phys. Rev. B 2001, 63, 245318

[62] Benchamekh, R. ; Gippius, N. A. ; Even, J. ; Nestoklon, M. O. ; Jancu, J. M. ; Ithurria, S. ; Dubertret, B. ; Efros, Al. L. ; Voisin, P. Physical Review B 2014, 89, 035307

[63] Sukkabot, W. Journal of Computational Electronics 2017, 16, 796-804

[64] Zakharov, O. ; Rubio, A. ; Cohen, M. L. Physical Review B 1995, 51, 4926-4930

[65] Mohr, M. ; Thomsen, C. Nanotechnology 2009, 20, 115707

[66] Kissinger, N. J. S. ; Jayachandran, M. ; Perumal, K. ; Raja, S. C. Bull. Mater. Sci. 2007, 30, 547551 
[67] Xu, Y. N. ; Ching, W. Y. Phys. Rev. B 1993, 48, 4335

[68] Burger, A. ; Henderson, D. O. ; Morgan, S. H. ; Silberman, E. Journal of Crystal Growth 1991, 109, 304-308

[69] Grimme, S. J. Comput. Chem. 2006, 27, 1787

[70] Grimme, S. ; Antony, J. ; Ehrlich, S. ; Krieg, H. J. Chem. Phys. 2010, 132, 154104

[71] Madelung, O. Semiconductors - Basic Data, Springer-Verlag, 5 Berlin Heidelberg, Marburg, 2nd edn, 1996, p. 185

[72] Parsons, R. B.; Wardzynski, W.; Yoffe, A. D. Royal Society 1961, 262, 1308

[73] Tasker, P. W. J. Phys. C: Solid State Phys. 1979, 12, 4977-4984

[74] Szemjonov, A. ; Tasso, M. ; Ithurria, S. ; Ciofini, I. ; Labat, F. ; Pauporté, T. Journal of Photochemistyr and Photobiology A 2019, 368, 182-189 